\title{
IS THERE A LINK BETWEEN A LOW LEVEL OF VITAMIN D AND MULTIPLE SCLEROSIS?
}

\author{
Dinu Cristian Popescu1,2, Dan Trofin¹, Orest Bolbocean ${ }^{1,2}$, Bogdan Ignat ${ }^{1,2}$, \\ Daniela-Marilena Trofin ${ }^{1,2}$ \\ ${ }^{1}$ The Neurology Clinic, The Rehabilitation Hospital, Iasi \\ 2 "Gr. T. Popa” University of Medicine and Pharmacy, Iasi
}

\begin{abstract}
Multiple Sclerosis is a neurological disorder consisting in autoimmune and inflammatory manifestations. Its evolution is characterized by axonal demyelination among the central nervous system.

A growing body of evidence suggests lately that vitamin D deficiency is a very common condition in Multiple Sclerosis. On the other hand, during the last years, several studies have shown a correlation between high vitamin D serum levels and a decreased risk either for developing this illness, or preventing its evolution towards disability. Vitamin $\mathrm{D}$ is currently being considered an important environmental factor for this disease, therefore being widely debated whether its supplementation would actually be benefic, and if yes, what results can be expected in such a therapeutic approach.

Our brief look upon some of the latest results in the field of research for this matter is intended to synthesize what is known at this moment and what future directions would be.
\end{abstract}

Keywords: vitamin D, multiple sclerosis

\section{INTRODUCTION}

Multiple Sclerosis (MS) is one of the most frequent demyelinating pathologies of the central nervous system (CNS), most often affecting young adults, causing disability by accumulation of deficits throughout manifestations referred to as "attacks" or "relapses".

The inflammatory component of MS targets the brain and the spinal cord, many genetic associations being investigated nowadays, as well as the possibility of slowing down the progression of the disease, especially by preventing the relapses.

In this context, the relationship between the sources of vitamin D (such as diet or sun light exposure) and the risk for MS is currently intensively debated.

Vitamin D is not only important for bone metabolism, but it is also responsible for immune regulation in T helper and dendritic cells. The implica- tions of vitamin $\mathrm{D}$ in neuro-inflammation, associated with the deficiency rate in MS patients, makes vitamin D supplementation, a therapeutic strategy worth to be considered. There are several results at this moment that reflect both a decrease of the inflammatory process, and the attenuation of disability progression. (1)

From environmental aspects of vitamin D deficit, to several studies on animal correspondent demyelinating pathology regarding vitamin D effects, and also taking into consideration the immune and genetic approaches, it becomes more obvious that vitamin $\mathrm{D}$ is starting to play an important role in MS management.

\section{ENVIRONMENTAL ASPECTS}

During the last years, the possible role of vitamin D as a modifiable environmental factor in the 
prevention or/and treatment of MS emerges as a promising therapeutic approach. (2)

Alimentation in specific areas where fish oil, a rich cholecalciferol source, is frequently consumed is known to lower the risk for MS exacerbations. (3)

The geographical distribution of MS, increasing with latitude in the both hemispheres, is a starting point to considering the link between the disease's prevalence and latitude. Thus, an etiology for a low level of vitamin D production in the organism has been considered to be the decreased quantum of ultraviolet light, with direct negative effect among immunomodulatory functions. (4)

Ultraviolet radiation, as involved in insufficient sun exposure resulting in low levels of vitamin D, affects the MS risk independently of the human leukocyte antigen HLA-DRB1*15 status, as mentioned in a population-based case-control study from Sweden (Bäärnhielm et al, 2012). The study involved 1013 cases of MS and 1194 controls, investigated by sun exposure habits, vitamin D level and HLA genotype, the associations between these conditions being performed by logistic regression (odds ratio, 95\% confidence intervals). The risk was increased in subjects with less sun exposure and also high for patients with 1,25-dihydroxyvitamin D [1,25-(OH)(2)D] level lower than $50 \mathrm{nM} / \mathrm{I}$. The correlation between sun light exposure and MS risk persisted even after an adjustment to the vitamin D status. (5)

A multicenter incidental case-control study, from Australia, suggests the independent role of sun exposure and vitamin D status in the risk of CNS demyelination (Lucas et al, 2011). 216 MS patients and 395 controls, from 4 Australian centers, were investigated in terms of sun exposure, high actinic skin damage and vitamin D serum level, suggesting that high levels of various moments' sun exposure are linked to a reduced risk for demyelinating events whereas a high serum vitamin D status is independently associated with a lower risk. (6)

Another population case-control study, this time in Tasmania (latitude 41-43 degrees S), attempting to determine a link between the 25-hydroxyvitamin D [25(OH)D] status and MS, has been conducted on $136 \mathrm{MS}$ cases, suggesting a high association between low 25(OH)D levels, reduced sun exposure and high disability (Expanded Disability Status Scale - EDSS above 3) (van der Mei et al, 2007). The authors suggest that determination of vitamin $\mathrm{D}$ degree of insufficiency might be benefic in MS patients, as therapeutically restoration of these levels can be integrated in the clinical management of MS preventing disability. (7)

Attempts to understand the pathogenesis of sex differences in the risk for MS, as well as the link to environmental factors with implications in this disease have been made by proving the lower incidence of MS and related disabilities in women with high circulation levels of 25(OH)D (Kragt et al, 2009). For this, measurements of serum concentrations of $25(\mathrm{OH}) \mathrm{D}$ and $(1,25(\mathrm{OH})(2) \mathrm{D})$ were performed, both in winter and summer, on $103 \mathrm{MS}$ patients and 110 healthy controls. As expected, the serum concentrations were higher during summer determinations, surprisingly in the women group a protective effect of higher $25(\mathrm{OH}) \mathrm{D}$ levels being observed. (8)

\section{MONTH OF BIRTH EFFECT}

We discussed above about the geographical distribution of MS as an environmental factor, dependent on latitude. We will next refer about a theory that was more and more debated over the last decade.

A month on birth effect refers to the risk of developing MS that seems to be increased for children born in spring and early summer (April, May and June) compared to the ones born in winter. This affirmation is valid especially for the northern hemisphere, and suggests that sun exposure of the pregnant woman during summer and early autumn is protective. On the other hand, a pregnancy that goes on during late autumn and dark and cold winter months carries a higher risk. In the both seasonrelated examples, the end of the second or the third trimester is the important time point referred to. $(9,10,11)$

The month of birth effect has been studied in especially in northern countries such as Sweden, Norway or Canada.

A population based study including results from over 42,000 patients with MS in high prevalent countries: Canada, Great Britain, Denmark and Sweden revealed that being born in May represents a higher risk for MS than being born in November. (Willer et al, 2005) According to the results, 9.1\% patients were born in May, compared to $8.5 \%$ in November. Odds ratio was also compared for May increased risk versus November, the highest being found in Scotland, Denmark and Canada. Altogether, MS patients from the northern hemisphere possessed a $13 \%$ higher risk if born in May compared to November. (9) 
There are also other populations the authors refer to, such as the Danish, where it is suggested that more MS patients seem to be born in March, April, May and June; as well another Swedish set of results suggesting March, May and even July. (9)

Also, another study from Norway (6,649 patients) reveals a higher MS risk in April (10), and another Swedish one, based on 9361 cases from the Swedish MS Registry, suggests an association between the risk for MS and the season of birth, referring to the period February-July in comparison to August-January. (11)

The moment of birth may be important in MS as there might be an interaction with the development of the central nervous system and the immune system. There is also possible that an association with latitude in early life is present, as it was observed in migrants from different risk regions. (9)

\section{VITAMIN D AND EXPERIMENTAL AUTOIMMUNE ENCEPHALOMYELITIS}

There are quite numerous reports throughout the last decade, suggesting studies on mice with experimental autoimmune encephalomyelitis (EAE), an animal correspondent for MS in humans, showing the involvement of vitamin D in inhibiting the progression of the disease. (12)

Several studies in EAE mice suggest a reduction of the severity of the disease after vitamin D administration, on the other hand, a lower vitamin D level means a risk for subsequent relapse. (13)

The over-representation of vitamin D receptor gene $b$ allele in Japanese MS patients suggests the susceptibility for the disease. In experiments on mice with EAE, 1,25-(OH)(2)D3 inhibits both induction and progression. In this particular case, interleukin 4 and transforming growth factor beta-1 synthesis was stimulated, and also influenced inflammatory cell trafficking and apoptosis. (3)

Natural immunoregulatory and anti-inflammatory functions have been also explored as a possibility of influencing MS through paracrine or autocrine cellular metabolism of $25(\mathrm{OH}) \mathrm{D}$ in peripheral tissues, with implications in both neural and immune functions in mice (Van Amerongen et al, 2004). Therefore, administration of $1,25-(\mathrm{OH})(2) \mathrm{D}$ in mice with experimental allergic encephalomyelitis showed both prevention and reduction of disease activity, suggesting a possible benefit for $25(\mathrm{OH}) \mathrm{D}$ therapeutically adjustments in MS patients, aiming suppression of disease activity and preventing long term complications, with second- ary benefit among fractures or muscle weakness. (14)

\section{ASSESSING IMMUNE RESPONSES AND GENETIC PERSPECTIVES}

Beside the latitudinal prevalence of MS, vitamin D-related genes might also be related to MS susceptibility, as well as the month of birth effect. $(4,15)$

It is known that Imunoglobulin-like transcript ILT3 and ILT4 are inhibitory receptors that are important in modulating immune responses and that several reports suggest that their expression might be affected by interferon. Waschbisch et al investigated the expression of ILT3 and ILT4 on immune cells from MS patients as well as in post-mortem brain tissue, and also the inducing of in vitro upregulation of these receptors by interferon beta alone or combined with vitamin D. Some of the main conclusions of this study were that ILT3 levels were found to be increased in demyelinating lesions in the postmortem brain, expression on monocytes of both ILT3 and ILT4 in the cerebrospinal fluid is higher than in peripheral blood, thus ILT3 and ILT4 show important involvement in immune response modulation in MS, influenced by interferon and vitamin D. (16)

Unbalanced 25(OH)D gene expression is likely to affect MS activity, as in another recent study, the number of gadolinium-enhancing lesions seems to be associated with 25(OH)D levels (Munger et al, 2014). By investigating the serum level of $25(\mathrm{OH}) \mathrm{D}$ and global gene expression profiles of patients staring interferon beta $-1 \mathrm{~b}$ after clinically isolated syndrome (with MRI assessment), the authors concluded that $25(\mathrm{OH}) \mathrm{D}$ is responsible for regulating immune modulatory processes in MS activity by gene-interaction network through vitamin D receptor, therefore an increase of $25(\mathrm{OH}) \mathrm{D}$ levels has as a result a decrease in the illness's activity (effects also enhanced by interferon beta1b). (17)

Genetic studies also suggest that there is a link between MS and vitamin D and therefore vitamin D3 supplementation has a protective role in this disease. The affirmation is based on the fact that genotypes of rs2248359 locus are associated with an increased risk of MS by regulating expression of the CYP24A1 gene. This gene encodes the enzyme involved in the degradation of 1,25-dihydroxyvitamin D3. (18)

Another recent study from Slovakia reveals data regarding the Fokl vitamin D receptor gene poly- 
morphism and its association with the risk of MS and disability progression in this disease. Cierny et al investigated $270 \mathrm{MS}$ patients and 303 healthy controls with Fokl single nucleotide polymorphisms in the vitamin D receptor, by polymerise chain reaction (PCR), concluding on no significant correlation between Fok1 SNP and the progression of the disability, however, a slight association between VDR SNP Fokl and MS risk in women $(\mathrm{p}=0.042)$ remaining possible and to be demonstrated in following studies. (19)

Vitamin D is involved in pathological pathways also involving neuromyelitis optica (NMO) or NMO spectrum disorder (NMOSD). A study that included 51 NMSOD patients (aquaporin 4-antibody positive) and 204 healthy controls, correlated $25(\mathrm{OH}) \mathrm{D} 3$ levels with the EDSS, annulized relapse rate (ARR) and time of blood sampling relative to attack. 25(OH)D3 levels were found to be low in NMOSD patients, the only notable result being the inverse relation between the 25(OH)D3 levels and EDSS, thus the question whether a low vitamin D level is a risk for NMO (predisposal or aggravating the condition) or it might be secondary to the neurological disability? (20)

\section{VITAMIN D BINDING PROTEIN}

As most of the circulating vitamin D metabolites are bound to vitamin D binding protein (DBP), Smolders et co attempted to prove an eventual association between DBP and MS comparing DBP concentrations of 29 RRMS patients and 30 controls, afterwards trying to make correlations of the DBP with D25(OH)D and 1,25- $(\mathrm{OH})(2) \mathrm{D}$ levels, as well as the effect of vitamin D3 supplementation in RRMS patients. No correlation between DBP, MS and vitamin D level was observed; after supplementation only $1,25(\mathrm{OH})(2) \mathrm{D}$ correlated passively with DBP, possible relevant finding for vitamin D metabolism. (21)

Another recent study, dealing with the involvement of DBP in MS, included $28 \mathrm{MS}$ patients and 24 healthy controls in which DBP and albumin plasma levels were measured. (Rinaldi et co, 2015) The results of the study revealed that the MS patients posses higher DBP levels and that interferon beta therapy does not influence DBP concentration; also no correlation between albumin levels and DBP were observed. (22)

Also, DBP levels in the cerebrospinal fluid can be predictive for the course of MS, as they seem to be lower during attacks and higher especially within the secondary progressive phase. (23)
There is a possibility that high levels of DBP are not benefic for recovery, an upregulated level of DBP being possible to represent a diagnostic marker for the progression of MS. (24)

\section{LOW VITAMIN D LEVELS - MS CORRELATIONS AND STRATEGIES}

A low level of Vitamin D in plasma influences the degree of disability measured on the EDSS, being directly proportional with a higher level of handicap, as revealed by a recent retrospective cohort analysis that included 181 patients (Thouvenot et al, 2014). The vitamin D levels were higher in relapse-remitting MS than in the progressive forms, but still in the first type of disease, a low level of vitamin D was associated with an EDSS below 4 (patients with $>20 \mathrm{ng} / \mathrm{ml}$ vit.D). (25)

A study originated from India, a country with vitamin $\mathrm{D}$ deficiency, suggests the presence of a lower level of $25(\mathrm{OH}) \mathrm{D}$ in MS patients, compared to matched controls and also an obvious lower level of vitamin $D$ in patients during attack than those in remission $(\mathrm{p}=0.001)$. (Pandit, Ramagopalan, Malli et al, 2013) The study was based on the assessment of the low level of 25(OH)D levels in 110 MS patients, out of which 63 patients in relapse and 77 in remission and 108 controls. (26)

Starting from the observation that in RRMS a low serum level is associated with a higher relapse risk, and that pregnant women also carry a risk for vitamin D deficit, Runia et al investigated vitamin $\mathrm{D}$ levels and their clinical correlations in MS patients both during pregnancy and postpartum. The study included 43 pregnant MS patients and 21 pregnant controls, investigated for $25(\mathrm{OH}) \mathrm{D}$ level at several moments before, during and after pregnancy period. A low level of $25(\mathrm{OH}) \mathrm{D}$ was not correlated to postpartum relapse, the compared values of the analysis between the two lots of patients being not very different, nevertheless, the higher serum concentrations of vitamin D being generally associated to a better health state. Therefore, the authors conclude about non-necessarily supplementation of vitamin D in pregnant MS patients, as the risk for relapse doesn't seem to be increased postpartum. (27)

It is well known that sun exposure or diets rich in fish lower the risk for MS, fish oil being an excellent source for vitamin $\mathrm{D}$, however, as a therapeutic approach for a beneficial supplementation, diet may just not be enough. (3) Increased levels of vitamin D metabolits can be useful in MS treat- 
ments, especially for SPMS patients, but not only. (24)

Some proposed dosages for vitamin D administration in MS patients are situated between the recommendations for 3000 International Units (IU) per day in order to achieve a serum level of 75-100 $\mathrm{nmol} / \mathrm{L}(30-40 \mathrm{ng} / \mathrm{mL})$, followed by $500-800 \mathrm{IU}$ per day to maintain these desired values that seem to be protective against relapses and the progression of the disease. (28)

The European Food Safety Authority recommends vitamin $\mathrm{D}$ administration according to age, varying from $1000 \mathrm{IU} /$ day $(25 \mu \mathrm{g} /$ day $)$ in children under 1 year old, 2000 UI until the age of 10 $(50 \mu \mathrm{g} /$ day $), 4000 \mathrm{IU} /$ day $(100 \mu \mathrm{g})$ in young adults and adults, and also during pregnancy and lactation period. (29)

\section{CONCLUSIONS}

Both genetic and environmental factors remain incriminated in the susceptibility for MS, in northern countries the risk for the disease being higher for those born in spring. (9)

Vitamin D deficiency is considered to be a risk factor for MS, as low plasmatic levels of 25(OH)D have been associated with a higher risk for developing the disease and a much more severe clinical deterioration towards disability. Knowing these facts, an early vitamin D treatment might be be- nefic in influencing the progression of disability in MS, $(22,25)$ as in studies on EAE it reduces the severity of symptoms and the progression of the disease. (13)

Apart from slowing down the disease's progression, it is possible that the positive effect of vitamin $\mathrm{D}$, consisting in the amelioration of the inflammatory process, would be benefic for both the relapses and by this, for the disease's evolution. (1)

Vitamin D acts as a powerful immunomodulator, preventing proliferation of activated $\mathrm{B}$ cells and improving recovery (as also demonstrated on EAE). Therapeutic supplementation may also prevent developing MS as well as its role as adjuvant to more specific therapies already available. $(3,30)$

A higher circulating level of $25(\mathrm{OH}) \mathrm{D}$ also seems to be linked to a reduced relapse risk and a slowing down of MS activity. Also, the way that DBP cerebrospinal levels correlates with the evolution of the disease, is still to be further investigated. (22)

More studies, some of them ongoing, during following years, will most likely provide information about both the most appropriate dosing of vitamin $\mathrm{D}$ as well as the transgenerational effects. (31)

The results will surely justify the financial costs, time and effort of the scientific research community, in the attempt of providing a significantly improvement in the lives of patients suffering from MS.

\section{REFERENCES}

1. Faridar A., Eskandari G., Sahraian M.A., et al. Vitamin D and multiple sclerosis: a critical review and recommendations on treatment, Acta Neurol Belg. 2012 Dec; 112(4):327-33;

2. Niino M., Fukazawa T., Kikuchi S., Sasaki H. Therapeutic potential of vitamin D for multiple sclerosis, Curr Med Chem. 2008; 15(5):499-505;

3. Hayes C.E. Vitamin D: a natural inhibitor of multiple sclerosis, Proc Nutr Soc. 2000 Nov; 59(4):531-5;

4. Niino M., Miyazaki Y., Fukazawa T., Kikuchi S. Vitamin D and latitude as environmental factors in multiple sclerosis, Nihon Rinsho. 2014 Nov; 72(11):1924-9;

5. Bäärnhielm M., Hedström A.K., Kockum I., et al. Sunlight is associated with decreased multiple sclerosis risk: no interaction with human leukocyte antigen-DRB1*15, Eur J Neurol. 2012 Jul; 19(7):955-62;

6. Lucas R.M., Ponsonby A.L., Dear K., et al. Sun exposure and vitamin $\mathrm{D}$ are independent risk factors for CNS demyelination, Neurology. 2011 Feb 8;76(6):540-8;

7. van der Mei I.A., Ponsonby A.L., Dwyer T., et al. Vitamin D levels in people with multiple sclerosis and community controls in Tasmania, Australia, J Neurol. 2007 May; 254(5):581-90;
8. Kragt J., van Amerongen B., Killestein J., et al. Higher levels of 25-hydroxyvitamin $D$ are associated with a lower incidence of multiple sclerosis only in women, Mult Scler. 2009 Jan;15(1):9-15;

9. Willer C.J., Dyment D.A., Sadovnick A.D. et al. Timing of birth and risk of multiple sclerosis: population based study, BMJ. 2005 Jan 15; 330(7483):120;

10. Torkildsen O., Aarseth J., Benjaminsen E. et al. Month of birth and risk of multiple sclerosis: confounding and adjustments, Ann Clin Transl Neurol. 2014 Feb; 1(2):141-4;

11. Salzer J., Svenningsson A., Sundström P. Season of birth and multiple sclerosis in Sweden, Acta Neurol Scand. 2010 Jul; 122(1):70-3;

12. Niino $M$. Vitamin $D$ and its immunoregulatory role in multiple sclerosis, Drugs Today (Barc). 2010 Apr; 46(4):279-90;

13. Mowry E.M. Vitamin D: evidence for its role as a prognostic factor in multiple sclerosis, J Neurol Sci. 2011 Dec 15; 311(1-2):19-22;

14. VanAmerongen B.M., Dijkstra C.D., Lips P., Polman C.H. Multiple sclerosis and vitamin D: an update, Eur J Clin Nutr. 2004 Aug; 58(8):1095-109;

15. Pakpoor J., Ramagopalan S. Evidence for an Association Between Vitamin D and Multiple Sclerosis, Curr Top Behav Neurosci. 2014 Dec 13. 
16. Waschbisch A., Sanderson N., Krumbholz M., et al. Interferon Beta and vitamin d synergize to induce immunoregulatory receptors on peripheral blood monocytes of multiple sclerosis patients, PLoS One. 2014 Dec 31;9(12):e115488;

17. Munger K.L., Köchert K., Simon K.C., et al. Molecular mechanism underlying the impact of vitamin $\mathrm{D}$ on disease activity of MS, Ann Clin Transl Neurol. 2014 Aug; 1(8):605-17;

18. Ramasamy A., Trabzuni D., Forabosco P., et al. Genetic evidence for a pathogenic role for the vitamin D3 metabolizing enzyme CYP24A1 in multiple sclerosis, Mult Scler Relat Disord. 2014 Mar; 3(2):211-219;

19. Čierny D., Michalik J., Kurča E., et al. Fokl vitamin D receptor gene polymorphism in association with multiple sclerosis risk and disability progression in Slovaks, Neurol Res. 2015 Apr; 37(4):301-8;

20. Min J.H., Waters P., Vincent A., et al. Low levels of vitamin D in neuromyelitis optica spectrum disorder: association with disease disability, PLoS One. 2014 Sep 11; 9(9):e107274;

21. Smolders J., Peelen E., Thewissen M., et al. Circulating vitamin D binding protein levels are not associated with relapses or with vitamin D status in multiple sclerosis, Mult Scler. 2014 Apr; 20(4):433-7;

22. Rinaldi A.O., Sanseverino I., Purificato C., et al. Increased Circulating Levels of Vitamin D Binding Protein in MS Patients, Toxins (Basel). 2015 Jan 13; 7(1):129-37;

23. Disanto G., Ramagopalan S.V., Para A.E., Handunnetthi L. The emerging role of vitamin $D$ binding protein in multiple sclerosis, J Neurol. 2011 Mar; 258(3):353-8;
24. Yang M., Qin Z., Zhu Y., Li Y., et al. Vitamin D-binding protein in cerebrospinal fluid is associated with multiple sclerosis progression, Mol Neurobiol. 2013 Jun; 47(3):946-56;

25. Thouvenot E., Orsini M., Daures J.P., Camu W. Vitamin D is associated with degree of disability in patients with fully ambulatory relapsing-remitting multiple sclerosis, Eur J Neurol. 2014 Dec 20;

26. Pandit L., Ramagopalan S.V., Malli C., et al. Association of vitamin D and multiple sclerosis in India, Mult Scler. 2013 Oct; 19(12):1592-6;

27. Runia T.F., Neuteboom R.F., de Groot C.J., et al. The influence of vitamin $D$ on postpartum relapse and quality of life in pregnant multiple sclerosis patients, Eur J Neurol. 2014 Nov 28,

28. Summerday N.M., Brown S.J., Allington D.R., Rivey M.P. Vitamin $D$ and multiple sclerosis: review of a possible association, J Pharm Pract. 2012 Feb; 25(1):75-84;

29. EFSA Panel on Dietetic Products, Nutrition and Allergies (NDA); Scientific Opinion on the Tolerable Upper Intake Level of vitamin D. EFSA Journal 2012;10(7):2813.[45pp.] doi:10.2903/j.efsa.2012.2813. Available online: www.efsa.europa.eu/efsajournal;

30. Brown S.J. The role of vitamin $D$ in multiple sclerosis, Ann Pharmacother. 2006 Jun; 40(6):1158-61;

31. Berlanga-Taylor A.J., Ramagopalan S.V. Vitamin $D$ and multiple sclerosis: what is the clinical impact?, Expert Opin Med Diagn. 2013 May; 7(3):227-9. 